\title{
Desafio terapêutico: hemorragia digestiva por angioectasia bulbar em paciente com infarto agudo do miocárdio recente
}

\author{
Therapeutic challenge: digestive hemorrhage for bulbar angioectasia in a patient with \\ recent acute myocardial infarction
}
Reto terapéutico: hemorragia digestiva por angioectasia bulbar en un paciente con infarto agudo de miocárdio reciente

Fernanda Ferradeira Latorre ${ }^{1}$, José Rafael Araújo e Costa ${ }^{1 *}$, Matheus Santana Luz ${ }^{1}$, Radmila Alessandra de Souza Oliveira ${ }^{1}$, Raquel Ida Ferreira ${ }^{1}$, Marcus Vinícius de Paula da Silva ${ }^{1}$.

\section{RESUMO}

Objetivo: Relatar o manejo de um paciente com infarto agudo do miocárdio recente que apresenta indicação absoluta da descontinuação da terapia antiplaquetária devido à hemorragia digestiva alta por angioectasia bulbar. Detalhamentos do caso: Paciente idosa, hipertensa, ex-tabagista, em uso de ácido acetilsalicílico, clopidogrel, atorvastatina, metoprolol e captopril após angioplastia primária com implante de stent devido a infarto agudo do miocárdio com supra desnivelamento do segmento ST, evoluiu com quadro de dor epigástrica em queimação, melena e adinamia. Endoscopia digestiva alta evidenciou angioectasia em bulbo duodenal com sangramento em porejamento. Ao hemograma, hemoglobina de $5,2 \mathrm{~g} / \mathrm{dL}$. A paciente obteve boa resposta à ablação endoscópica da angioectasia, recebendo alta com suspensão da terapia antiplaquetária. Considerações finais: A despeito da ausência de dados na literatura, esse trabalho tem por objetivo relatar um quadro de difícil manejo sobre o ideal momento para se reintroduzir dupla antiagregação plaquetária, apesar do benefício de tais drogas em pacientes submetidos à intervenção coronariana percutânea, como a redução do risco de recorrência do infarto, nos casos de evidente risco de sangramento em que o paciente apresenta indicação absoluta da descontinuação da terapia antiplaquetária, faz-se necessário a individualização da indicação desse tratamento farmacológico.

Palavras-chave: Angioectasia bulbar, Hemorragia digestiva alta, Terapia antiplaquetária.

\section{ABSTRACT}

Objective: To report a picture of challenging management of a patient with recent acute myocardial infarction that has an absolute indication of discontinuation of antiplatelet therapy due to upper digestive hemorrhage due to bulbar angioectasis. Details of the case: Elderly, hypertensive patient, ex-smoker, using acetylsalicylic acid, clopidogrel, atorvastatin, metoprolol e captopril after primary angioplasty with stent implantation due to acute myocardial infarction with ST-segment elevation, evolved with burning epigastric pain, melena and adamia. Upper gastrointestinal endoscopy showed angioectasis in the duodenal bulb with bleeding in bleeding. At hemogram, hemoglobin of $5.2 \mathrm{~g} / \mathrm{dL}$. The patient had a good response to endoscopic angioectasis ablation and was discharged with suspension of antiplatelet therapy. Final considerations: Despite the lack of data in the literature, this study aims to report a difficult to manage situation about the ideal time to reintroduce double antiplatelet therapy, despite the benefit of such drugs in patients who have undergone percutaneous coronary intervention, such as reducing the risk of infarction recurrence, in cases of evident risk of bleeding in which the patient has an absolute indication of discontinuation of antiplatelet therapy, it is necessary to individualize the indication for this pharmacological treatment.

Key words: Bulio angioectasis, High digestive bleeding, Antiplatelet therapy.

${ }^{1}$ Faculdade de Medicina de Barbacena (FAME), Barbacena - MG. *E-mail: joserafaelsamonte@hotmail.com SUBMETIDO EM: 2/2021 


\section{RESUMEN}

Objetivo: Informar un cuadro de manejo desafiante de un paciente con infarto agudo de miocardio reciente que tiene una indicación absoluta de interrupción del tratamiento antiagregante plaquetario debido a hemorragia digestiva alta por angioectasia bulbar. Detalle del caso: Anciano, hipertenso, exfumador, en tratamiento con ácido acetilsalicílico, clopidogrel, atorvastatina, metoprolol y captopril tras angioplastia primaria con implante de stent por infarto agudo de miocardio con elevación del segmento ST, evolucionado con dolor epigástrico ardiente, melena y adamia. La endoscopia digestiva alta mostró angioectasia en el bulbo duodenal con sangrado. Enel hemograma, hemoglobina de 5,2 g / dL. El paciente tuvo buena respuesta a la ablación endoscópica de angioectasias y fue dado de alta con suspensión de la terapia antiagregante. Consideraciones finales: A pesar de la ausencia de datos en la literatura, este estudio tiene como objetivo reportar una situación de difícil manejo sobre el momento ideal para reintroducir la doble antiagregación, a pesar del beneficio de estos fármacos en pacientes que han sido sometidos a intervencionismo coronario percutáneo, como la reducción del riesgo de recurrencia del infarto, en los casos de evidente riesgo de hemorragia en los que el paciente tenga una indicación absoluta de interrupción de la terapia antiagregante, es necesario individualizar la indicación de este tratamiento farmacológico.

Palabras clave: Angioectasia buliogénica, Sangrado digestivo alto, Terapia antiplaquetaria.

\section{INTRODUÇÃO}

A Hemorragia Digestiva Alta (HDA) é uma causa comum de admissão hospitalar com níveis de mortalidade global em torno de $10 \%$, independentemente da sua etiologia específica. Essa entidade clínica é definida como sangramento de lesões localizadas no trato gastrointestinal acima do ângulo de Treitz, sendo que a lesão de angioectasia duodenal é uma causa incomum e de difícil diagnóstico. A apresentação clínica é variável e, por isso, a avaliação inicial é de fundamental importância no diagnóstico, bem como na definição dos critérios de gravidade e no manejo do paciente (STANKE PF, et al., 2019).

A angioectasiagastrointestinal é uma malformação arteriovenosa, cuja etiologia não é bem elucidada, que consiste em pequenos vasos anormais, dilatados e tortuosos, com paredes finas e diâmetro menor que 10 $\mathrm{mm}$ dentro da camada mucosa e submucosa do intestino, sendo frequentemente mais prevalente no intestino grosso (SAMI SS, et al., 2013). Nesse sentido, a angioectasia intestinal gera uma fragilidade local que eventualmente resulta em hemorragias no trato gastrointestinal, com manifestações variadas, como anemia, fadiga e até mesmo enterorragia (MAGALHÃES RT, et al., 2020).

Essa patologia é classificada em 3 tipos, baseada nas diferentes malformações arterio-vasculares (MAV's): Tipo I, com maior incidência no cécum e cólon direito; a Tipo II, mais frequente no intestino delgado e em pacientes jovens, sendo, mais extensas e de provável origem congênita, onde classifica-se a lesão do presente caso; e a Tipo III, que podem acometer qualquer parte do trato gastrointestinal (DALL'INHA VN e RIBAS JC, 2010; JACKSON CS e STRONG R, 2017). Pacientes portadores de angioectasia gastrointestinal em uso de terapia combinada de aspirina e clopidogrel são de difícil manejo terapêutico, visto que apresentam elevado risco hemorrágico.

Em seu último posicionamento, a Sociedade Brasileira de Cardiologia orienta que a realização da dupla antiagregação plaquetária (DAP) seja realizada por um mínimo de 12 meses em pacientes submetidas a intervenção coronária percutânea (ICP) após síndrome coronariana aguda (SCA), podendo ser modificada para um tempo mínimo de 6 meses em caso de risco aumentado de sangramento. Contudo, o manejo adequado ao paciente é individual. Em pacientes com risco de sangramento maior do que a de um evento trombótico, a descontinuação da terapia antiplaquetária deve ser considerada (DALL'INHA VN e RIBAS JC, 2010; SERRANO JRCV, et al., 2019).

Este trabalho tem por objetivo relatar um quadro de difícil manejo de HDA por angioectasia bulbar em paciente com infarto agudo do miocárdio há 2 meses que apresenta indicação absoluta da descontinuação da terapia antiplaquetária, sendo que não há dados na literatura sobre o momento ideal para a reintrodução desses agentes em tal situação. 


\section{DETALHAMENTO DO CASO}

Trata-se de uma paciente do sexo feminino, 71 anos, que procurou atendimento em unidade de emergência devido a queixa de dor epigástrica em queimação, insidiosa, progressiva, contínua e intensa. Queixou-se, ainda, de sangramento digestivo baixo tipificado como melena e de adinamia. Quanto aos antecedentes pessoais, relatou ser hipertensa, ex-tabagista e ter sido submetida à angioplastia primária com implante de stent convencional em ramo marginal esquerdo (ME), devido a infarto agudo do miocárdio com supra desnivelamento do segmento ST em parede inferior, há 2 meses. Na ocasião, recebeu alta hospitalar em uso de ácido acetilsalicílico $100 \mathrm{mg} /$ dia, clopidogrel $75 \mathrm{mg} /$ dia e atorvastatina $40 \mathrm{mg} / \mathrm{dia}$, metoprolol 100 $\mathrm{mg} /$ dia e captopril $50 \mathrm{mg} / \mathrm{dia}$, os quais relatou realizar uso contínuo.

Do ponto de vista gastrointestinal, afirmava diagnóstico prévio de angioectasia estável no bulbo duodenal com proposta de seguimento não invasivo. Ao exame físico encontrava-se em regular estado geral, lúcida e bem orientada, agitada, acianótica, anictérica, afebril, normotensa. À ausculta cardíaca, ritmo regular e taquicardíaco, com bulhas normofonéticas. À ausculta pulmonar, evidentes crepitações finas em bases. Abdome globoso e doloroso à palpação profunda em região epigástrica. Diante do quadro álgico, a paciente foi submetida à endoscopia digestiva alta, a qual evidenciou esofagite erosiva grau B de Los Angeles e mucosa hipocorada, bem como angioectasia em bulbo duodenal com coágulo facilmente retirável com instilação de água, evoluindo com sangramento do tipo porejamento.

Quanto aos exames laboratoriais, os componentes alterados foram Hemoglobina 5,2 $\mathrm{g} / \mathrm{dL}$ (valor de referência mulheres: $>12 \mathrm{~g} / \mathrm{dl}$ ) e Creatinina $2,85 \mathrm{~g} / \mathrm{dL}$ (valor referência entre 0,6 e 1,2mg/dl). Como conduta, a paciente foi submetida à ablação endoscópica da angioectasia bulbar com gás argônio (30W), sem intercorrências. Em vista dos níveis hematimétricos foi realizada transfusão de dois concentrados de hemácias. Ademais, iniciou-se hidratação venosa e omeprazol $40 \mathrm{mg} / \mathrm{dia}$, suspendendo o uso da aspirina 100 $\mathrm{mg} / \mathrm{dia}$, do clopidogrel75 mg/dia e dos anti-hipertensivos: metoprolol100mg/dia e captopril $50 \mathrm{mg} / \mathrm{dia}$. A paciente evoluiu com boa resposta ao tratamento, recebendo alta com proposta de avaliação ambulatorial do retorno da terapia antiplaquetária em momento oportuno.

A Figura 1 demonstra o bulbo duodenal anatômico, com mucosas lisas e íntegras, sendo evidenciado angioectasia sem sangramento ativo (Foto A). Realizada ablação da mesma com gás argônio (30W, 1,5 L) com êxito e sem intercorrências (Fotos B, C e D). Segunda porção duodenal sem alterações.

Figura 1 - Etapas da ablação endoscópica da angioectasia bulbar.

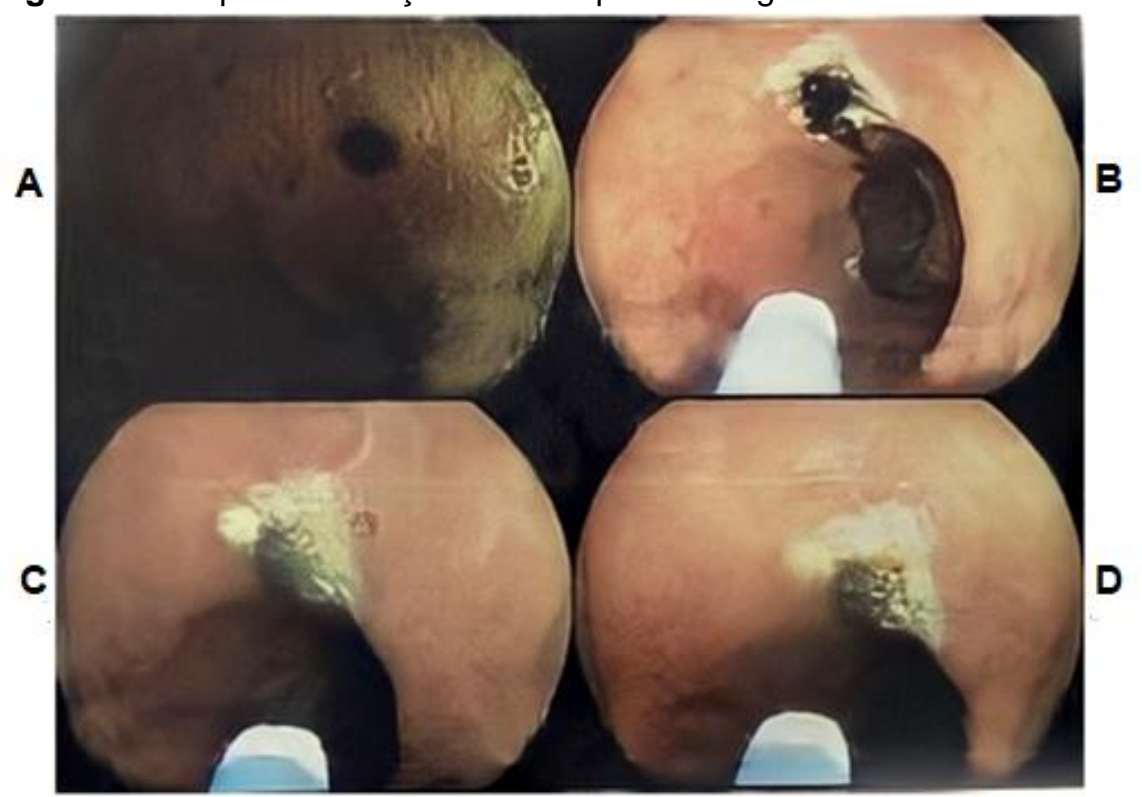

Legenda: A: Bulbo duodenal - angioectasia. B: Ablação com gás argônio. C: Bulbo duodenal- Angioectasia. D: Ablação com gás argônio.

Fonte: Costa JRA, et. al., 2021. 


\section{DISCUSSÃO}

As angiodisplasias ou angioectasias caracterizam-se por anormalidades vasculares, nas quais ocorrem ectasias de vasos sanguíneos submucosos (DALL'INHA VN e RIBAS JC, 2010; SALTZMAN JR, et al., 2017). Tratam-se de veias dilatadas e tortuosas, de paredes finas, revestidas por endotélio associado ou não à pequenas quantidades de músculo liso, frequentemente encontradas no trato gastrointestinal, com maior acometimento no intestino grosso (SALTZMAN JR, et al., 2017; CLOUSE RE, et al., 1985).

Apesar de poderem estar presentes ao nascimento ou serem desenvolvidas como parte de síndromes hereditárias, as angioectasias frequentemente são adquiridas ao longo da vida, todavia com etiologia pouco compreendida (SALTZMAN JR, et al., 2017). Acometem, principalmente, pacientes após a 6 ${ }^{\underline{a}}$ década de vida e, por se tratar de uma doença oligossintomática, consiste em um achado incidental de endoscopia digestiva alta em cerca de $40 \%$ dos casos (SALTZMAN JR, et al., 2017).

Cerca de $6 \%$ dos casos de hemorragias digestivas baixas (HDB) de caráter autolimitado tem como causa as angiodisplasias do intestino delgado. Aproximadamente $40-60 \%$ dos pacientes apresentam mais de uma angiodisplasia, predominantemente encontradas na mesma porção do trato gastrointestinal (SAURIN JC, et al., 2019; JAECKLE T, et al., 2008).

O acometimento do bulbo duodenal, como no caso descrito, é mais raro podendo ser uma causa importante de sangramento gastrointestinal com grandes consequências hemodinâmicas ao paciente caso não haja um devido manejo (CLOUSE RE, et al., 1985; HOLMES DR e SORAJJA P, 2019). Alguns fatores se associam ao risco de desenvolvimento e de sangramento das angioectasias, tais como doença renal crônica, coagulopatias e estenose aórtica (MAGALHÃES RT, et al., 2020).

A angioplastia transluminal percutânea é uma intervenção minimamente invasiva no campo da hemodinâmica, que consiste na desobstrução de artérias através do implante de stents, sobretudo após um evento de síndrome coronariana aguda. Nessa situação a terapia antitrombótica tende a ser uma parte importante para o sucesso do procedimento de revascularização, pois visa a prevenção de eventos cardiovasculares indesejáveis periprocedimento, como a ocorrência de trombose em stents, e a longo prazo, como a recorrência do infarto agudo do miocárdio (SERRANO JRCV, et al., 2019; LINCOFF AM, et al., 2020; SMITH KJ, et al., 2003).

Assim, é fortemente recomendado a terapia antiplaquetária dupla para todos os pacientes submetidos à intervenção coronariana percutânea - como foi o caso da paciente do referido trabalho-, através da combinação de ácido acetilsalić́lico (AAS) e de um bloqueador do receptor P2Y12 (clopidogrel, por exemplo) por pelo menos 12 meses. É recomendado o tratamento por 36 meses adicionais aos pacientes que obtiverem sucesso diante do uso da terapia em questão (LINCOFF AM, et al., 2020; EIKELBOOM JW, et al., 2006).

Entretanto, é válido ressaltar que os regimes antitrombóticos atuais têm o potencial de resultar em casos de sangramento gastrointestinal, aumentando o risco para a ocorrência de hematêmese, melena ou enterorragia, ou ainda de exacerbar um sangramento crônico, sendo responsáveis por aproximadamente $15 \%$ de todos os eventos de alguma perda sanguínea periprocedimento (HOLMES DR e SORAJJA P, 2019).

No caso apresentado, a paciente encontrava-se em uso da DAP com AAS e clopidogrel há 2 meses, devido a colocação de stent farmacológico após infarto do miocárdio, quando apresentou o evento hemorrágico. Para os pacientes que apresentam sangramento gastrointestinal devido à terapia antiplaquetária, existem morbidade e mortalidade significativas atribuíveis a este evento (TAYLOR JE, et al., 1991). Por isso, não se pode deixar de avaliar e de eliminar os fatores desencadeadores do sangramento. ADAP induz um estado de hipocoagulabilidade devido à redução da função plaquetária, apesar destas se apresentarem com contagem dentro do valor de referência (LINCOFF AM, et al., 2020; FERRARI E, et al., 2005).

Desta forma, o uso do AAS e clopidrogrel apresentaram-se como fator indutor para o sangramento da angiodisplasia previamente presente no bulbo da paciente. Sabe-se que o AAS e outros anti-inflamatórios não esteroidais são capazes de bloquear a enzima ciclooxigenase (COX). Assim, no trato gastrointestinal há 
inibição da proteção de sua mucosa de revestimento, bem como o aumento da secreção ácida, podendo levar a erosões ou a hemorragia (BATLOUNI M, et al., 2010). Além disso, o bloqueio da COX também inibe a produção do tromboxano $\mathrm{A} 2$, que é um potente estimulador da agregação plaquetária, favorecendo a ocorrência de sangramentos.

Já o clopidogrel, e outros bloqueadores do receptor P2Y12 de plaquetas, tem a propriedade de inibir a agregação plaquetária a partir da ligação do receptor de difosfato de adenosina plaquetária ao complexo glicoproteína Ilb-Illa (TAYLOR JE, et al., 1991). Portanto, o uso de tais medicamentos está associado ao aumento no risco de sangramento gastrointestinal; entretanto com magnitude não é bem elucidada.

O uso da DAP por seis meses a um ano em pacientes com síndrome coronariana aguda que sofreram intervenção coronariana percutânea reduz o risco de recorrência do infarto, de trombose do stent e de acidente vascular encefálico. O benefício da intervenção é ainda maior nos casos de intervenção coronariana percutânea complicada, que inclui colocação de 3 ou mais stents, tratamento de 3 ou mais lesões ou 3 coronárias distintas, tratamento de bifurcação com 2 stents, comprimento do stent maior que $60 \mathrm{~mm}$ ou tratamento de lesão crônica (COSTA F, et al., 2019; FREITAS AH, et al., 2009).

Por outro lado, o risco-benefício da terapêutica antiplaquetária deve ser cuidadosamente avaliado em pacientes com maior risco de sangramento. Apesar do benefício notório da DAP na síndrome coronariana aguda e no procedimento de intervenção coronariana percutânea, pacientes com alto risco de sangramento, como a paciente apresentada neste artigo, não se beneficiam com a redução da mortalidade almejada por essa terapêutica, devido a maior ocorrência de eventos hemorrágicos, necessitando da descontinuação da terapia medicamentosa para a hemostasia (COSTA F, et al., 2019; HOLMES DR e SORAJJA P, 2019).

De fato, estudos mostram que os mecanismos associados a sangramentos em paciente após angioplastia primária com implante de stent pode relacionar-se com o aumento da mortalidade, com causa multifatorial (HOLMES DR e SORAJJA P, 2019; EIKELBOOM JW, et al., 2006).

Dentre as consequências do evento hemorrágico destaca-se a ocorrência de hipercoagulabilidade pela interação entre as plaquetas e a cascata de coagulação (LANE DA, et al., 2005). Além disso, dados experimentais sugerem que o aumento da síntese e liberação de eritropoietina em resposta à anemia causada pelo sangramento pode sustentar um estado pró-trombótico sistêmico por ativar e induzir o inibidor do ativador de plasminogênio (PAI-1). Níveis elevados de PAI-1 estão relacionados à redução da atividade fibrinolítica e, por conseguinte, o aumento do risco de doenças cardiovasculares e da trombose (TAYLOR JE, et al., 1991; SMITH KJ, et al., 2003).

Entretanto, diante de sangramentos, a descontinuação prematura de terapias antitrombóticas leva a um aumento do risco de eventos isquêmicos miocárdicos recorrentes, incluindo trombose de stent, visto que o próprio sangramento agudo pode levar à ativação das plaquetas e ao início da cascata de coagulação para hemostasia, aumentando potencialmente 0 risco de trombose na ausência de terapia antiplaquetária (HOLMES DR e SORAJJA P, 2019).

Em caso de sangramento gastrointestinal agudo, a decisão de interromper o AAS, o bloqueador do receptor P2Y12 ou outra terapia antitrombótica deve ser individualizada para cada paciente, equilibrando a probabilidade e as consequências para uma ação de evento trombótico ou hemorrágico (HOLMES DR e SORAJJA P, 2019). O tratamento do sangramento digestivo causado pela angioectasia requer a avaliação e o tratamento definitivo por via endoscópica (MAGALHÃES RT, et al., 2020). Vários métodos têm sido utilizados para hemostasia, como: injeção de soluções na submucosa (soro fisiológico, esclerosantes, vasoconstrictores) e métodos térmicos (cautérios monopolar e bipolar, gás argônio (FREITAS AH, et al., 2009).

A técnica de coagulação de plasma de argônio, método terapêutico de escolha no caso, tem sido descrita pela literatura como método de baixo custo, seguro e popular, devido à sua facilidade de uso. Trata-se de um método em que é usada energia de alta frequência transmitida ao tecido por gás ionizado e que vem sendo usada para tratamento de uma grande variedade de lesões com sangramento, incluindo a angiodisplasia (SALTZMAN JR, et al., 2017). 
Nesse sentido, para a referida paciente, o uso da DAP foi suspenso até o tratamento endoscópico definitivo da angiodisplasia. A paciente recebeu alta com a proposta de retorno da DAP em tempo oportuno, uma vez que não há consenso na literatura em relação ao momento ideal para reintrodução desses agentes. Com relação ao sangramento após intervenção coronariana, o uso de inibidores da bomba de prótons está associado a uma incidência significativamente menor de sangramento gastrointestinal dentro dos primeiros 30 dias após o procedimento de intervenção percutânea. Em termos de prevenção secundária, os inibidores da bomba de prótons podem reduzir o risco de recorrência do trato gastrointestinal superior (HOLMES DR e SORAJJA P, 2019; CHIN MW, et al., 2007).

Apesar do benefício do uso da DAP em pacientes vítimas de síndrome coronariana aguda, em casos de incontestável risco de sangramento, como apresentado neste relato, não há serventia no objetivo da redução da mortalidade através dessa abordagem (COSTA F, et al., 2019). Nesse sentido, ainda que devam ser feitos esforços para continuar a terapia antiplaquetária, a paciente foi conduzida com a suspensão da DAP, visto que Ihe traria maiores danos, dada a magnitude do sangramento.

Para pacientes que foram submetidos à intervenção coronariana com stents farmacológicos que apresentarem sangramento gastrointestinal, especialmente se o implante de stent coronário tiver sido realizado recentemente, é razoável mudar de terapia antiplaquetária dupla para monoterapia por seis meses, no caso de síndromes coronárias agudas. Em pacientes para os quais foi determinado que a terapia antiplaquetária absolutamente precisa ser descontinuada, não há dados sobre o momento ideal para a reintrodução desses agentes ou das doses apropriadas que devem ser usadas (HOLMES DR e SORAJJA P, 2019).

Dessa forma, a abordagem foi feita pela resolução da angioectasia através do tratamento endoscópico definitivo e, posteriormente, considerada possibilidade de retornar com o tratamento da DAP, para que haja profilaxia de futuros eventos isquêmicos. Portanto, este caso é um desafio terapêutico único, bem como chama a atenção para a necessidade de individualizar a indicação do tratamento antiagregante plaquetário, guiando-se pelo risco de sangramento da paciente.

\section{REFERÊNCIAS}

1. BATLOUNI M. Anti-Inflamatórios Não Esteroides: Efeitos Cardiovasculares, Cérebro Vasculares e Renais. Arquivos Brasileiros de Cardiologia, 2010; 94(4): 556-563

2. CHIN MW, et al. Predictive and protective factors associated with upper gastrointestinal bleeding after percutaneous coronary intervention: a case-control study. The American Journal of Gastroenterology, 2007; 102: 2411.

3. CLOUSE RE, et al. Angiodisplasia como causa de sangramento gastrointestinal superior. Archives of Internal Medicine, 1985; 145: 458-61.

4. COSTA F, et. al. Dual Antiplatelet Therapy Duration Based on Ischemic and Bleeding Risks After Coronary Stenting. Journal of the American College of Cardiology, 2019; 73 (7): 741-754.

5. DALL'INHA VN, RIBAS JC. Angiodisplasia do Intestino Delgado: Revisão da Literatura e Novas Perspectivas. Arquivos Catarinenses de Medicina, 2010; 39(4): 80-84

6. EIKELBOOM JW, et al. Adverse impact of bleeding on prognosis in patients with acute coronary syndromes. Circulation, 2006; 114: 774-782.

7. FERRARI E, et al. Coronary syndromes following aspirin withdrawal: a special risk for late stent thrombosis. Journal of the American College of Cardiology, 2005; 45(3): 456-459.

8. FREITAS AH, et al. Novo método para tratamento da angiodisplasia de cólon. Revista Brasileira de Coloproctologia, 2009; 29(4): 485-488.

9. HOLMES DR, SORAJJA P. Gastrointestinal bleeding in patients undergoing percutaneous coronary intervention. UpToDate, 2019.

10. JACKSON CS, STRONG R. Gastrointestinal Angiodysplasia Diagnosisand Management. Gastrointestinal Endoscopy Clinics of North America, 2017; 27: 51-62.

11. JAECKLE T, et al. Acute gastrointestinal bleeding: value of MDCT. Abdominal Imaging, 2008;33(3):285-93.

12. LANE DA, et al. Directing thrombin. Blood, 2005; 106: 2605-2612.

13. LINCOFF AM, et al. Antiplatelet agents in acute ST-elevation myocardial infarction. UpToDate; 2020 Disponível em: https://www.uptodate.com/contents/acute-st-elevation-myocardial-infarction-antiplatelet-therapy\#! 
14. MAGALHÃES RT, et. al. Angiodisplasia intestinal em paciente com doença renal crônica: relato de caso. Revista Eletrônica Acervo Saúde, 2020; 48: 1-6.

15. SALTZMAN JR, et al. Angiodysplasia of the gastrointestinal tract. UpToDate, 2017. Disponível em: https://www.uptodate.com/contents/angiodysplasia-of-the-gastrointestinal-tract\#!

16. SAMI SS, et al. Review article: gastrointestinal angiodysplasia - pathogenesis, diagnosis and management. Alimentary Pharmacology\&Therapeutics. 2014; 39(1): 15-34.

17. SAURIN JC, et al. Treatment of digestive angiectasia: time for prospective, randomized, therapeutic studies. Endoscopy International Open, 2019; 7(12): E1778-E1779.

18. SERRANO JRCV, et al. Statement on Antiplatelet Agents and Anticoagulants in Cardiology. Journal of Brazilian Society of Cardiology, 2019; 113(1): 109-111.

19. 19)SMITH KJ, et al. The cardiovascular effects of erythropoietin. Cardiovascular Research. 2003; 59: $538-548$.

20. STANKE PF, et al. Lesão de dieulafoyembulbo duodenal. Relatos de Casos Cirúrgicos. 2019; 3: 2247.

21. TAYLOR JE, et al. Erythropoietin and spontaneous platelet aggregation in haemodialysis patients. Lancet.1991; 338: 1361-1362. 